\title{
Resecciones colorrectales segmentarias en pacientes con colitis ulcerosa y displasia o adenocarcinoma
}

\author{
Alberto Vilar-Tabanera ${ }^{1}$, Ana Puerta V. ${ }^{1}$, Pedro Abadía B. ${ }^{1}$ y Javier Die ${ }^{1}$
}

\section{Segmental colectomy in patients with colitis-associated neoplasia or dysplasia}

Introduction: The historical management for patients with ulcerative colitis and displasia or adenocarcinoma associated was to perform a total proctocolectomy, what cause important morbidity and affect patient's quality of life. Materials and Method: A retrospective review about 5 patients with a segmental colectomy due to dysplasia or adenocarcinoma is done. Results: The median age at diagnosis of ulcerative colitis and cancer was 56 and 62 years respectively. Disease time evolution was between 1 and 13 years. The patients were followed up for a median of 57 months. During the follow-up evaluation, 1 patient was found to have dysplasia. Conclusion: Segmental colectomy could be a safe option in clinically stable patients, few years of diagnosis and with and a suitable follow up.

Key words: ulcerative colitis; colorectal adenocarcinoma; segmental colectomy.

\section{Resumen}

Introducción: El tratamiento estándar para los pacientes con colitis ulcerosa y displasia o adenocarcinoma de colon ha sido la proctocolectomía total, lo que conlleva una morbilidad y una reducción en la calidad de vida significativa. Materiales y Método: Se hace un análisis retrospectivo de 5 pacientes con colitis ulcerosa a los que se realiza una resección segmentaria por displasia o adenocarcinoma. Resultados: La mediana de edad al diagnóstico de colitis ulcerosa y de la neoplasia fue de 56 y 62 años respectivamente. El tiempo de evolución de la enfermedad fue de 1 a 13 años. La mediana de seguimiento postoperatorio fue de 57 meses apareciendo en uno de los pacientes un nuevo foco de displasia. Conclusiones: En determinados pacientes seleccionados, las resecciones segmentarias podrían ser una opción segura si tienen buen control de la enfermedad, escasa actividad inflamatoria, pocos años de evolución y que puedan realizar un adecuado seguimiento posterior.

Palabras clave: colitis ulcerosa; adenocarcioma colorrectal; colectomía segmentaria.

\section{Introducción}

El cáncer colorrectal (CCR) es una de las principales causas de muerte en pacientes con colitis ulcerosa (CU). Los pacientes con enfermedades inflamatorias intestinales, tienen un riesgo significativamente mayor al de la población general para desarrollar cáncer colorrectal ${ }^{1}$. El $30 \%$ de los pacientes con CU van a precisar una intervención quirúrgica a lo largo de su vida, siendo las principales indicaciones de cirugía el fracaso del tratamiento médico y la aparición de displasia o $\mathrm{CCR}^{2}$.

La asociación entre CU y CCR fue sugerida por primera vez en 1925, posteriormente en un estudio de 1971 el riesgo de desarrollo de CCR se estimó en el $5 \%$ y el $40 \%$ a los 10 y a los 25 años de enfermedad respectivamente. El riesgo de desarrollo de CCR aumenta a partir de los 8 años del diagnóstico, jugando un papel importante el ambiente creado por la inflamación crónica en el desarrollo de la patogénesis del cáncer colorrectal. Los pacientes con CCR y CU son pacientes más jóvenes que los pacientes con CCR esporádico, más frecuentemente son tumores múltiples y con subtipos histológicos mucinoso o en anillo de se$10^{3}$. Sin embargo, estudios más recientes sugieren que la incidencia actual de CCR en pacientes con CU es menor de lo esperado, mostrando Jess et al.,
'Departamento de

Coloproctología del Hospital Universitario Ramón y Cajal. Madrid, España.

Recibido el 17 de octubre de 2019 y aceptado para publicación el 11 de diciembre de 2019.

Correspondencia a:

Dr. Alberto Vilar T. alb.vilartabanera@gmail.com 
una incidencia de $0,4 \%$ de CCR a los 10 años y entre un $1,1 \%$ y un $5,3 \%$ a los 20 años ${ }^{4}$.

Un reciente estudio realizado en Turquía, que analiza la prevalencia de CCR y displasia en 801 pacientes con CU, muestra una prevalencia de CCR de $0,7 \%$ y de displasia de $0,85 \%$, y una incidencia acumulada de CCR de $0,3 \%$ a los 10 años, $1,3 \%$ a los 20 años y de $5,9 \%$ a los 30 años ${ }^{5}$. El registro nacional sueco con datos de 5.886 pacientes con CU muestra que únicamente el $0,8 \%$ presenta cáncer de recto después de realizarse una colectomía subtotal con preservación del recto ${ }^{6}$. En la Tabla 1 se resumen los principales estudios que analizan la incidencia de CCR y CU.

Este descenso en la incidencia se debe entre otros motivos a la mejora en el tratamiento médico de estos enfermos, con la supresión de la inflamación subclínica utilizando aminosalicitatos, inmunosupresores y fármacos biológicos.

El tratamiento actualmente reconocido para el CCR en pacientes con CU es la proctocolectomía total con creación de un reservorio ileal o con realización de ileostomía terminal. Sin embargo, estas técnicas no están exentas de morbilidad y mortalidad. En vista de estas complicaciones y el empobrecimiento de la calidad de vida, surge el planteamiento de la realización de resecciones parciales o segmentarias colorrectales, aunque la literatura para apoyar este tipo de manejo es escasa ${ }^{7,8}$.

El conocimiento de los factores de riesgo para el desarrollo de CCR es imprescindible para poder seleccionar los pacientes que precisan un seguimiento más estrecho, incluso para poder seleccionar los pacientes que podrían beneficiarse de resecciones parciales y seguimiento posterior. Los principales factores de riesgo son:

- Pancolitis: La extensión anatómica de la colitis y el grado de inflamación son factores de riesgo independientes, presentando alto riesgo los pacientes con pancolitis, riesgo moderado los pacientes con colitis izquierda y bajo riesgo los pacientes con proctitis?.

- Edad de diagnóstico: Cuanto antes sea el diagnóstico de CU mayor es el riesgo de desarrollo de CCR. Comparando con los pacientes diagnosticados por encima de los 40 años, el riesgo relativo (RR) cuando el diagnóstico se realiza antes de los 19 años es de 43,8 (95\% CI: 27,2-70,7), en cambio cuando el diagnóstico se realiza entre los 20 y los 39 años el RR baja a 2,65 (95\% CI: $1,97-3,56)^{4}$.

- Duración de la enfermedad: el tiempo de evolución está en relación con un aumento de riesgo de $\mathrm{CCR}^{10}$.
- Colangitis esclerosante primaria (CEP): Se ha estimado el riesgo acumulado de desarrollo de CCR en pacientes con CEP a los 10 años de $9 \%$, a los 20 años $31 \%$ y tras 25 años de $50 \%$, siendo muy superior al resto de pacientes con $\mathrm{CU}^{11}$.

- Historia familiar de $\mathrm{CCR}^{12}$.

- Displasia: Cuando en el seguimiento se descubre una displasia de bajo grado, el riesgo de desarrollo de CCR es 9 veces mayor, apareciendo un CCR sincrónico o una displasia de alto grado en el $19 \%$ de los pacientes en los que se realiza colectomía y desarrollan una neoplasia un 30\%$50 \%$ a los 5 años. La displasia de alto grado supone un riesgo de CCR sincrónico de $43 \%{ }^{13}$.

Nuestro objetivo es realizar un análisis de nuestra experiencia y el seguimiento posterior en pacientes con CU y CCR o displasia en los que hemos realizado resecciones segmentarias.

\section{Materiales y Método}

Se identificaron 289 pacientes en seguimiento por CU en el Hospital Ramón y Cajal en enero de 2018. De ellos, 34 pacientes han sido sometidos a cirugía por cáncer o displasia, en 5 de los casos se realizó una resección segmentaria por diagnóstico preoperatorio de displasia o adenocarcinoma. Se ha realizado un análisis retrospectivo de estos pacientes, analizando la morbilidad y el desarrollo posterior de CCR o displasia tras la cirugía.

El análisis de los datos estadísticos se realizó con el programa de datos estadístico SPSS $20^{\mathrm{a}}$ ed $\AA$. Las variables categóricas se expresan en porcentaje. Las variables cuantitativas con distribución normal se expresan como media y desviación estándar. Se describen variables cuantitativas con distribución no normal con la mediana y el rango intercuartílico.

\section{Consideraciones éticas}

Los autores declaran que para esta investigación no se han realizado experimentos en seres humanos ni en animales. Los autores declaran que en este artículo se ha preservado en todo momento el anonimato del paciente. El consentimiento informado no se solicitó para la publicación de estos casos, porque en el presente artículo no se publican datos personales que permita identificar al paciente.

\section{Resultados}

De los 5 pacientes incluidos, 4 son varones. La mediana de edad al diagnóstico de $\mathrm{CU}$ fue de 56 
Tabla 1. Riesgo de desarrollo de cáncer colorrectal en pacientes con colitis ulcerosa

\begin{tabular}{|lllccccc|}
\hline Referencia & Año & País & $\mathbf{1 0}$ años & $\mathbf{1 5}$ años & $\mathbf{2 0}$ años & $\mathbf{2 5}$ años & $\mathbf{3 0}$ años \\
Lennard-Joses et al & 1990 & Reino Unido & & $3 \%$ & $5 \%$ & & \\
Langholz et al & 1992 & Dinamarca & & & & $3,1 \%$ \\
Stewenius et a & 1995 & Suecia & & $2 \%$ & $3 \%$ & $4 \%$ \\
Wandall et al & 2000 & Dinamarca & & & $5,3 \%$ & $10,1 \%$ & $18,4 \%$ \\
Eaden et al & 2001 & Reino Unido & $1,6 \%$ & & $8,3 \%$ & & $6,1 \%$ \\
Hata et al & 2003 & Japón & $0,5 \%$ & & $4,1 \%$ & $2,1 \%$ \\
Winther et al & 2004 & Dinamarca & $0,4 \%$ & & $1,1 \%$ & $7,5 \%$ \\
Lakatos et al & 2006 & Hungría & $0,6 \%$ & & $5,4 \%$ & $2,7 \%$ \\
Söderlund et al & 2009 & Suecia & $1 \%$ & & $1,5 \%$ & \\
Jess et al & 2012 & USA & $<1 \%$ & $0,4-2 \%$ & $1-5,3 \%$ & & $5,9 \%$ \\
\hline Gülşen et al & 2019 & Turquía & $0,3 \%$ & & $1,3 \%$ & & \\
\hline
\end{tabular}

años (RIQ 44-60), la mediana de edad al diagnóstico de neoplasia fue a los 62 años (RIQ 46,5-67,5). El tiempo de evolución de la enfermedad entre ambos diagnósticos es de 1 a 13 años, con una mediana de 6 años. Ninguno de los pacientes tiene antecedentes familiares de CCR, ni padecían colangitis esclerosante primaria.

En todos los casos la actividad inflamatoria se encontraba localizada únicamente en colon descendente. Los pacientes con adenocarcinoma o displasia de colon ascendente no presentaron actividad inflamatoria en la pieza quirúrgica. Ninguno de los pacientes había sufrido brotes graves de su enfermedad siendo controlados adecuadamente con tratamiento oral con salicilatos en tres pacientes y en otro caso salicilatos en espuma rectal. Un paciente se encontraba con tratamiento con esteroides orales que se suspendieron previamente a la intervención quirúrgica programada, posterior a la misma continuó con tratamiento con mesalazina.

Los resultados de anatomía patológica fueron de displasia de alto grado en 3 pacientes y en dos casos adenocarcinoma. Se realizaron tres hemicolectomías derechas, una resección anterior baja y una colectomía izquierda, realizando en todos los casos anastomosis primaria.

Como complicaciones postquirúrgicas, hubo un caso de hemoperitoneo que precisó reintervención con buena evolución posterior.

El tiempo medio de seguimiento postoperatorio tuvo una mediana de 57 meses, con un mínimo de 12 meses y un máximo de 9 años, durante el cual se han realizado colonoscopias de control. Uno de los pacientes en la última colonoscopia realizada en enero de 2019 presenta varias zonas con displasia de bajo grado, por lo que se le ha propuesto la realización de una proctocolectomía total. En los otros 4 pacientes no se han encontrado nuevos focos de displasia.

\section{Discusión}

En nuestra serie la edad de diagnóstico de $\mathrm{CU}$ fue mayor de 40 años (56 años), el tiempo de evolución fue en la mayoría de los casos corto (entre 1 y 13 años), sin presencia de pancolitis grave y sin diagnóstico previo de CEP ni antecedentes familiares de CCR. La actividad inflamatoria se localizaba únicamente en colon descendente y los pacientes no habían sufrido brotes graves durante su evolución. El conjunto de todos estos factores permite poder seleccionar a los pacientes que se pueden beneficiar de una colectomía parcial frente a otros pacientes con una gran actividad inflamatoria, brotes graves y muchos años de evolución de la enfermedad que se beneficiarán de realizar una proctocolectomía total.

Durante un periodo de seguimiento medio de 57 meses, únicamente hemos encontrado un caso de displasia de bajo grado tras la cirugía inicial, por lo que en casos seleccionados parece ser una opción segura realizar resecciones segmentarias disminuyendo la morbilidad y con una mejor calidad de vida que con la realización de proctocolectomía total.

Khan et al. ${ }^{14}$, mostraron unos resultados similares, comparando las características de 35 pacientes a los que realizaban una proctocolectomía total y 24 pacientes a los que realizaban una resección colóni- 
ca parcial. Los pacientes con resecciones parciales tenían mayor edad, más comorbilidades y CU menos activa. Durante el seguimiento de 7 años, no presentaron adenocarcinomas ni displasias metacrónicas ninguno de los 24 pacientes.

Los resultados de Lindberg et al. ${ }^{15}$, también defienden que resecciones colónicas limitadas en pacientes con CU, mejoran la calidad de vida de los pacientes y pueden ser una alternativa a la proctocolectomía total sin aumentar el riesgo de muerte por CCR.

A pesar de que se trata de una serie pequeña y que la bibliografía que propone este tipo de manejo del paciente con displasia o adenocarcinoma asociado a CU es limitada, parece que la adecuada selección de los pacientes y la individualización del tratamiento puede permitirnos evitar proctocolectomías totales en determinados pacientes con un adecuado seguimiento posterior e informando al paciente de las posibilidades y del riesgo de realizar una colectomía segmentaria.

Como principal limitación, disponemos de un tamaño muestral pequeño, para poder extraer conclusiones, sin embargo, la bibliografía muestra una disminución de la incidencia de cáncer en pacientes con CU, unido a la mejora de las técnicas diagnósticas, endoscópicas y de seguimiento de los pacientes puede permitirnos realizar resecciones parciales realizando un correcto seguimiento posterior. Sería necesario realizar un metaanálisis para extraer conclusiones.

\section{Conclusiones}

Las resecciones segmentarias de colon podrían ser una opción segura en determinados pacientes seleccionados con un diagnóstico de CU tardío, buen control de la enfermedad, actividad inflamatoria local, pocos años de evolución y que puedan realizar un adecuado seguimiento posterior, informando al paciente de la posibilidad de desarrollo de nuevas displasias y adenocarcinomas colorrectales durante el seguimiento.

\section{Responsabilidades éticas}

Protección de personas y animales. Los autores declaran que para esta investigación no se han realizado experimentos en seres humanos ni en animales.

Confidencialidad de los datos. Los autores declaran que en este artículo no aparecen datos de pacientes.

Conflictos de interés: no hay.

\section{Bibliografía}

1. Wanders LK, Dekker E, Pullens B, Bassett P, Travis SP, East JE. Cancer risk after resection of polypoid dysplasia in patients with longstanding ulcerative colitis: a meta-analysis. Clin GastroenterolHepatol. 2014;12:756-64.

2. Laine L, Kaltenbach T, Barkun A, McQuaid KR, Subramanian V, Soetikno R. SCENIC International Consensus Statement on Surveillance and Management of Dysplasia in Inflammatory Bowel Disease. Gastroenterology 2015;148:639-51.e28.

3. Chambers WM, Warren BF, Jewell DP, Mortensen NJ. Cancer surveillance in ulcerative colitis. Br J Surg. 2005;92:92836.

4. Jess T, Simonsen J, Jørgensen KT, Pedersen BV, Nielsen NM, Frisch M. Decreasing risk of colorectal cancer in patients with inflammatory bowel disease over 30 years. Gastroenterology 2012;143:375-81.e1; quiz e13-4.

5. Ünal NG, Özütemiz Ö, Tekin F, Turan İ, Osmanoğlu N. Colorectal cancer and dysplasia risk in patients with ulcerative colitis at a tertiary referral center in Turkey. Turkish J Gastroenterol. 2019;30:118-9.

6. Abdalla M, Landerholm K, Andersson P, Andersson RE, Myrelid P. Risk of Rectal Cancer After Colectomy for Patients With Ulcerative Colitis: A National Cohort Study. Clin Gastroenterol Hepatol. 2017;15:1055-60.e2.

7. Krugliak Cleveland N, Ollech JE, Colman RJ, Rodríguez D, Hirsch A, Cohen RD, et al. Efficacy and follow-up of segmental or subtotal colectomy in patients with colitisassociated neoplasia. Clin Gastroenterol Hepatol. 2019; 17:205-6.

8. Kornbluth A, Sachar DB. Ulcerative colitis practice guidelines in adults (update): American College of
Gastroenterology, Practice Parameters Committee. Am J Gastroenterol. 2004;99:1371-85.

9. Jess T, Rungoe C, Peyrin-Biroulet L. Risk of colorectal cancer in patients with ulcerative colitis: a meta-analysis of population-based cohort studies. Clin Gastroenterol Hepatol. 2012;10:639-45. doi: 10.1016/j.cgh.2012.01.010. Epub 2012 Jan 28.

10. Brackmann S, Andersen SN, Aamodt G, Langmark F, Clausen OP, Aadland E, et al Relationship between clinical parameters and the colitis-colorectal cancer interval in a cohort of patients with colorectal cancer in inflammatory bowel disease. Scand J Gastroenterol. 2009;44:46-55.

11. Soetikno RM, Lin OS, Heidenreich PA, Young HS, Blackstone MO. Increased risk of colorectal neoplasia in patients with primary sclerosing cholangitis and ulcerative colitis: a meta-analysis. Gastrointest Endosc. 2002;56:48-54. 
12. Askling J, Dickman PW, Karlén P, Broström O, Lapidus A, Löfberg R, et al. Family history as a risk factor for colorectal cancer in inflammatory bowel disease. Gastroenterology 2001;120:135662 .

13. Thomas T, Abrams KA, Robinson RJ, Mayberry JF. Meta-analysis: cancer risk of low-grade dysplasia in chronic ulcerative colitis. Aliment Pharmacol Ther. 2007;25:657-68.

14. Khan N, Cole E, Shah Y, Paulson EC. Segmental resection is a safe oncological alternative to total proctocolectomy in elderly patients with ulcerative colitis and malignancy.
Diseases of the Colon \& Rectum 2018;61:713-8.

15. Lindberg J, Stenling R, Palmqvist R, Rutegård J. Surgery for neoplastic changes in ulcerative colitis-can limited resection be justified? Outcome for patients who underwent limited surgery. Colorectal Dis. 2006;8:551-6. 